\section{A Colour Atlas of Ocular Tumours}

By M. A. Bedford. Pp. 78, illustrated. Wolfe Medical

Publications, London, 1979. $£ 12.00$.

The Wolfe Medical Publications Atlases are well known and the Colour Atlas of Ocular Tumours is a useful but specialized new addition to the series. The page size $(260 \mathrm{~mm} \times 190$ $\mathrm{mm}$ ) larger than most previous Wolfe Atlases complements this particular subject very well. A strong shiny cloth binding is in keeping with the high standard of production of the Wolfe Atlas series.

Michael Bedford is an expert in ocular tumours and it is fitting that his extensive knowledge of this ophthalmological sub-speciality should be summarized in this Atlas. The 224 colour photographs amply cover the clinical appearance of most ocular and eyelid tumours, with a very brief descriptive text for each photograph. A number of pages have a rather crowded layout where the text of one photograph appears to run into the text of an adjoining one.

$A$ Colour Atlas of Ocular Tumours will be a very useful reference and read for all ophthalmic surgeons in training and might also prove useful for specialized oncologists and radiotherapists to see their specimens well illustrated in situ. The absence of a detailed text and histology of course reduces the effectiveness of the Atlas for more experienced ophthalmic surgeons which is a pity as the excellent photographs deserve a wide readership.

However, it is well understood that the Wolfe Atlas series do not claim to be comprehensive publications on their subjects and a Colour Atlas of Ocular Tumours may be considered a worthy easy-to-read addition to the Wolfe Atlas series.

\section{A Colour Atlas of Peripheral Vascular Diseases.}

By W. F. Walker. Pp. 112, illustrated. Wolfe Medical Publications, London, 1980. $£ 15.00$.

Vascular disease in its various forms accounts for nearly one half of the deaths in our so-called civilized world. Peripheral arterial diseases are responsible for much suffering and loss of work, as well as being the indication for the great majority of amputations today. Varicose veins and their complications are the most frequent cause of referral to general surgical out-patients and, indeed, most hospitals have to set up special clinics to deal with these patients. There is therefore a wealth of important pathological and clinical conditions which are of intense interest to today's medical student, and practitioners. Mr William Walker, who is Consultant Surgeon at Ninewells Hospital, Dundee, has produced a magnificent colour atlas of both common and unusual conditions of the arteries, veins and lymphatic system. His material ranges over acute and chronic ischaemia, gangrene, cerebrovascular disease, aneurysms, blood vessel tumours, trauma, varicose veins, deep vein thrombosis and its consequences, lymphoedema and tumours of the lymphatic vessels, illustrating their pathology, clinical features and angiography. Like the other volumes in this well known series of medical atlases, this serves as a magnificent supplement to the standard textbooks on vascular surgery. Medical students and postgraduate surgical trainees will benefit greatly from a careful study of this atlas - the next best thing to seeing the patients themselves.

\section{A Colour Atlas of Tumour Histopathology.}

By N. F. C. Gowing. Pp. 272, illustrated. Wolfe Medical Publications, London, 1980. $£ 35.00$.

This Atlas by one of this country's foremost tumour histopathologists is a very welcome addition to the series. Being directed primarily at trainee pathologists it is arranged by site, and each organ or system has a section devoted to it. Most tumours, both common and rare, arising in each site are illustrated, mainly by photomicrographs of $\mathbf{H} \&$ E-stained sections. Where helpful, appropriate special stains, photo- $\frac{2}{\mathbb{Q}}$ graphs or gross specimens and occasional electron micro- $\varrho$ graphs are illustrated. A number of proliferative lesions. $\subset$ which may be mistaken for malignant tumours are also $\Rightarrow$ included.

As the title implies, this is purely an atlas and the text is confined to clear descriptive captions to each photograph, many of which include brief clinical details or points of $\overline{\bar{N}}$ differential diagnosis.

Professor Gowing has clearly drawn from his vast collection of material to produce this book and is to be congratulated on its comprehensiveness and the very high quality of the illustrations both at low and high magnifications.

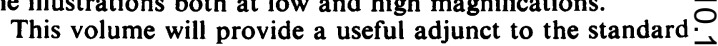
text books for the trainee pathologist but will also be of $\vec{\overrightarrow{ }}$ interest to all who deal with tumours, both clinicians and pathologists. It has already been helpful in solving one or twotricky slide seminar problems!

It is a pity that its price may deter those for whom it is 3 . primarily intended from buying their own copy but it will be a if most useful addition to the book shelf of every histopathology? laboratory. The Development of the Infant and Young Child. Normal and $\frac{\infty}{0}$
Abnormal.

By R. S. Illingworth. 7th edn. Pp. xi +320 , illustrated. 음 Churchill Livingstone, Edinburgh, London and New York, 1980. $£ 10.00$

It is no surprise to find that this well known book has entered its 7 th edition since it first appeared 20 years ago. The subject $\Phi$ is one to which the author has himself made outstandigg 3 personal contributions. The style is authoritative and charąteristically crisp and readable. The subject matter is concerng with the assessment of both physical and mental developmest $\vec{\theta}$ and it is supported by extensive data from the literature. The influence of prenatal and environmental factors are described. and the methodology of assessment is given in some detart. Sub-sections are devoted to cerebral palsy and the evaluation of suitability for adoption. Throughout, the author is at pains to indicate the limitations of the available techniques, $\bar{D}$ the need for interpretation of the data obtained and the recognition of the errors which can occur. This is a book to be $\mathbb{D}$ read and enjoyed by those learning about and working with children. It is commended as an invaluable text for hospital $\overline{0}$ and community doctors, and nurses involved in paediatric $\exists$ work and for the enquiring student.

\section{Diagnostic Electron Microscopy of Tumours}

By Feroze N. Ghadially. Pp. ix +251 , illustrated. Butterworths, London and Boston, 1980. £32.00.

The limitations of light microscopy in the diagnosis of $\bar{z}$ certain tumours are a recurrent source of frustration to practising histopathologists and their clinical colleagues. Where available, ultrastructural examination can be of 3 considerable help in identifying the nature of apparently 0 anaplastic carcinomas, soft tissue sarcomas, and several other groups of tumours which share similar histological appear- $D$ ances. Professor Ghadially's new book aims to clarify this area of surgical pathology and to introduce the principles of tumour ultrastructure, mainly by dealing with a series of $\sigma$ typical diagnostic problems.

After a brief introductory section on those aspects of $E$. M. technique relevant to diagnostic work, there is a section on $\mathrm{\omega}$ the ultrastructure of tumours in general and the differences $\sigma$ between normal and neoplastic cells. Some basic knowledge of normal cellular structure is assumed in this book which can be regarded as complementary to the author's earlier $\mathbb{D}$ volume Ultrastructural Pathology of the Cell. However, for those with such background knowledge, the current volume is self-contained. 